\title{
Macrophage polarization mediated by chitooligosaccharide (COS) and associated osteogenic and angiogenic activities
}

Xiuhong Huang, Meng Chen, Haoming Wu, Yanpeng Jiao*, Changren Zhou

Department of Materials Science and Engineering, Jinan University, Guangzhou, 510632, China

*To whom correspondence should be addressed:

E-mail: tjiaoyp@jnu.edu.cn (Yanpeng Jiao)

Tel and fax: $+86-20-85223271$

Pages: 4

\section{Contents}

Figure $\mathrm{S} 1$ S2

Figure S2. S3

Figure S3. S4 

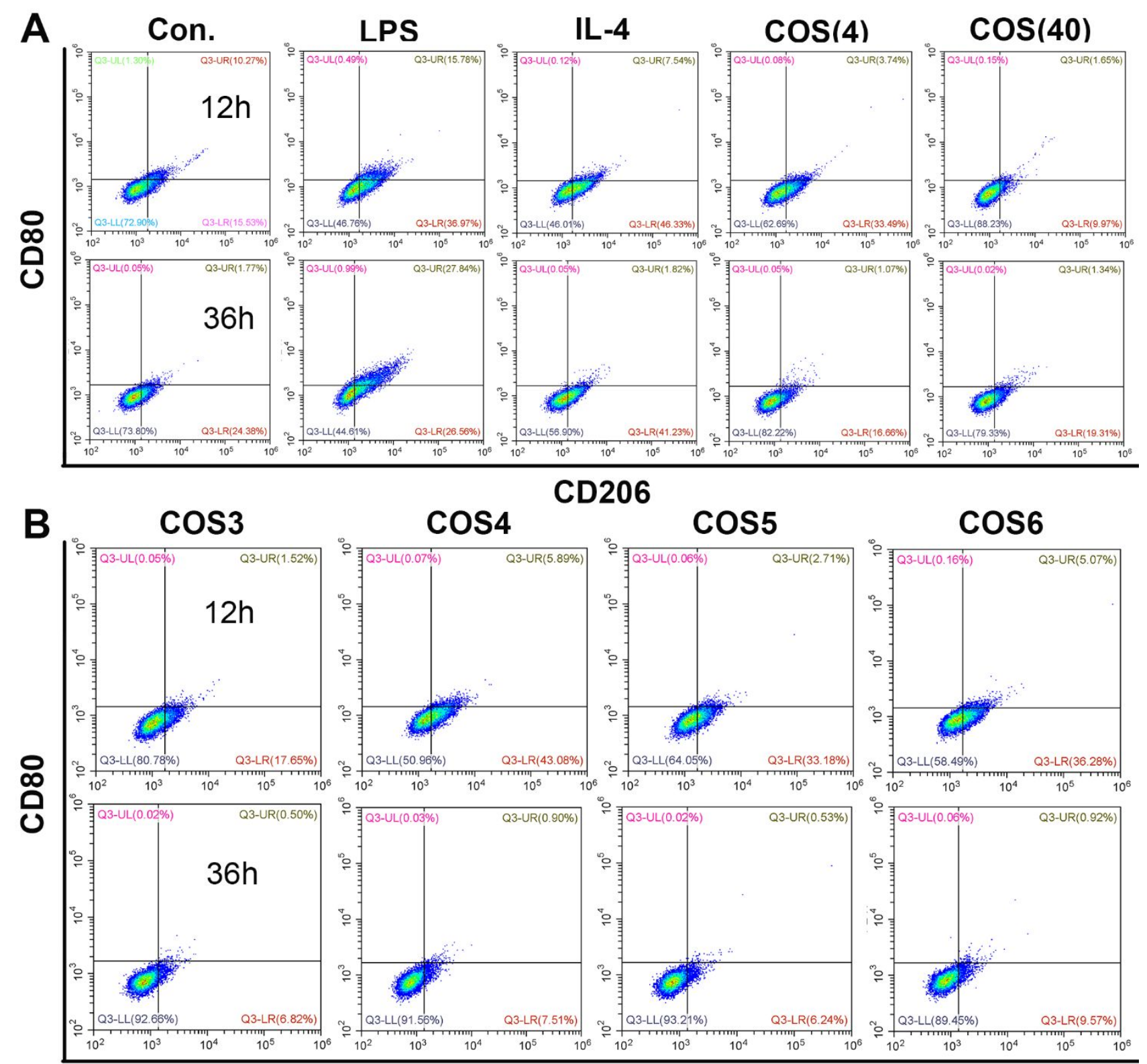

\section{CD206}

Figure S1. Expression of cell surface markers on RAW 264.7 macrophages cultured in COS media with different concentrations for $12 \mathrm{~h}$ and $36 \mathrm{~h}(\mathrm{~A})$, and with different DDs for $12 \mathrm{~h}$ and $36 \mathrm{~h}(\mathrm{C})$. Detections were carried out by FCM. 
A

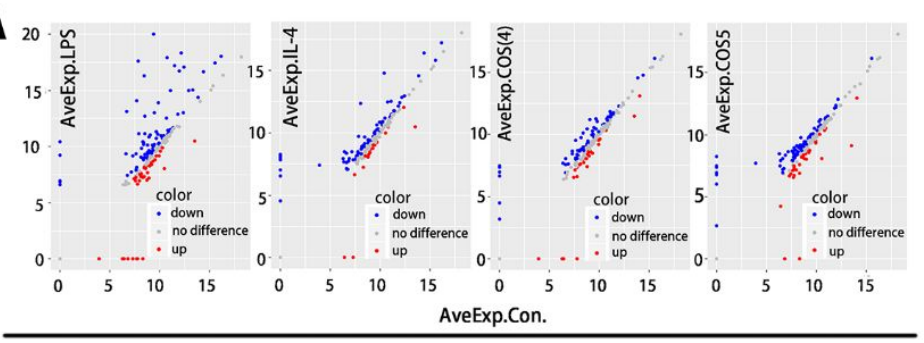

$\mathrm{B}_{1}$

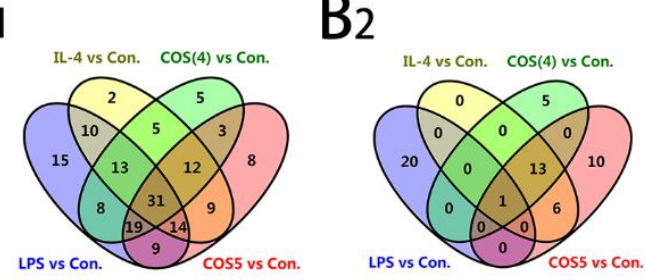

$\mathrm{C}_{1}$
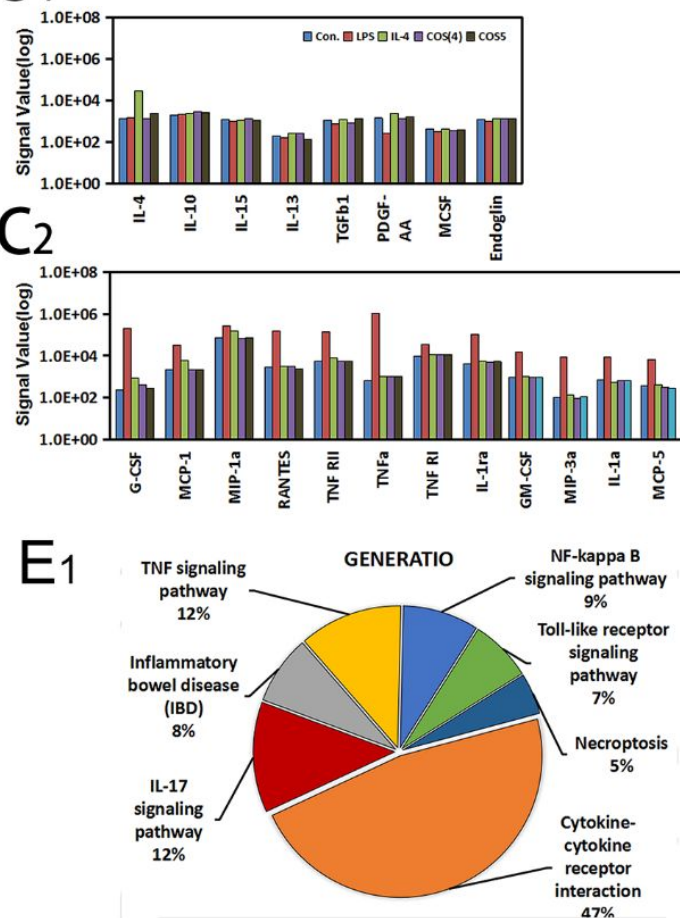

E2 P13k-Altsignaling

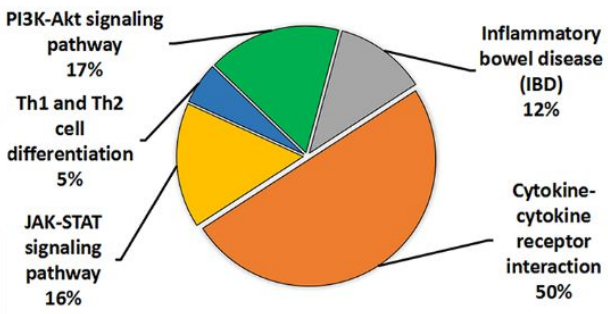

$D_{1}$

cell activation involved in immune response wound healing production of molecular mediator of immune response regulation of adaptive immune response regulation of JAK-STAT cascade regulation of immune effector process regulation of mononuclear cell proliferation positive regulation of cell activation positive regulation of cytokine production positive regulation of response to external stimulus

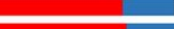
regulation of inflammatory response cell chemotaxis
cytokine-mediated signaling pathway positive regulation of cell migration

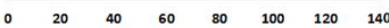

$D_{2}$

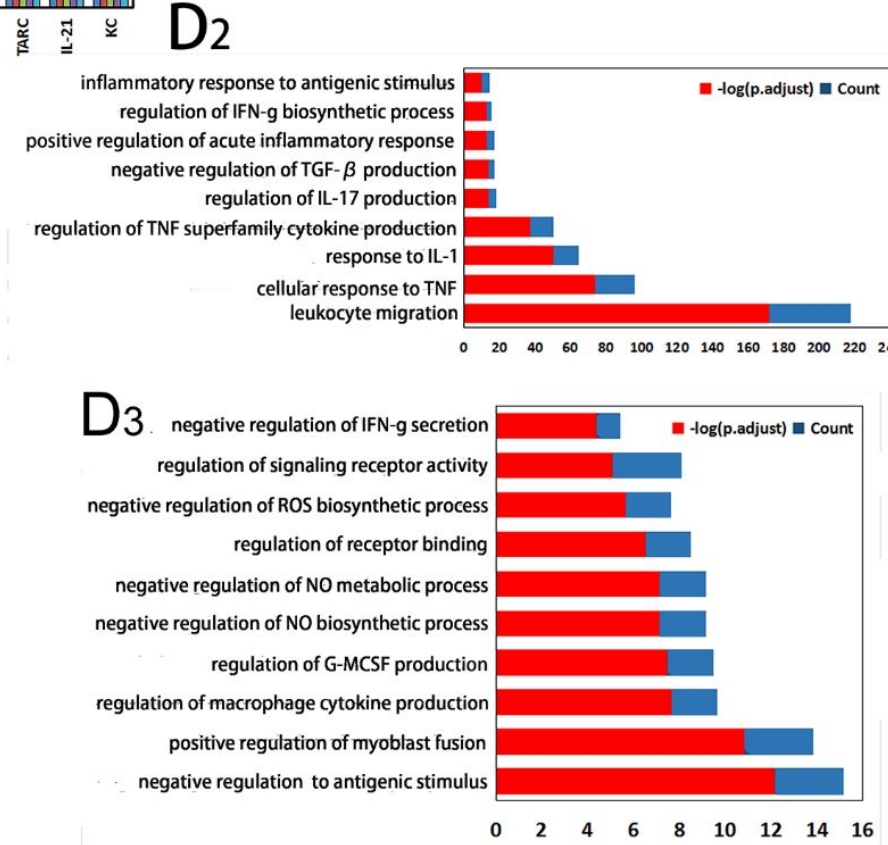

Figure S2. Differential proteins including upregulated (red) and downregulated (blue) in macrophages stimulated by LPS, IL-4, COS(4), and COS5, compared to the unstimulated cells (A). Venn diagram of total number of differential proteins in cells treated with LPS, IL-4, COS(4), and COS5 and overlaps $\left(\mathrm{B}_{1}\right)$. Similar Venn diagram of total number of enriched biological processed in cells and overlaps $\left(\mathrm{B}_{2}\right)$. Signals of anti-inflammatory $\left(C_{1}\right)$ and pro-inflammatory $\left(C_{2}\right)$ cytokines in macrophages following indicated treatments. The overlap of enriched inflammation-related biological processes of macrophages following COS5 and IL-4 treatments $\left(D_{1}\right)$, following LPS treatment $\left(D_{2}\right)$, and following COS5 treatment $\left(D_{3}\right)$. Inflammation-related pathway analysis of macrophages treated with LPS $\left(E_{1}\right)$ and $\operatorname{COS} 5\left(E_{2}\right)$. 

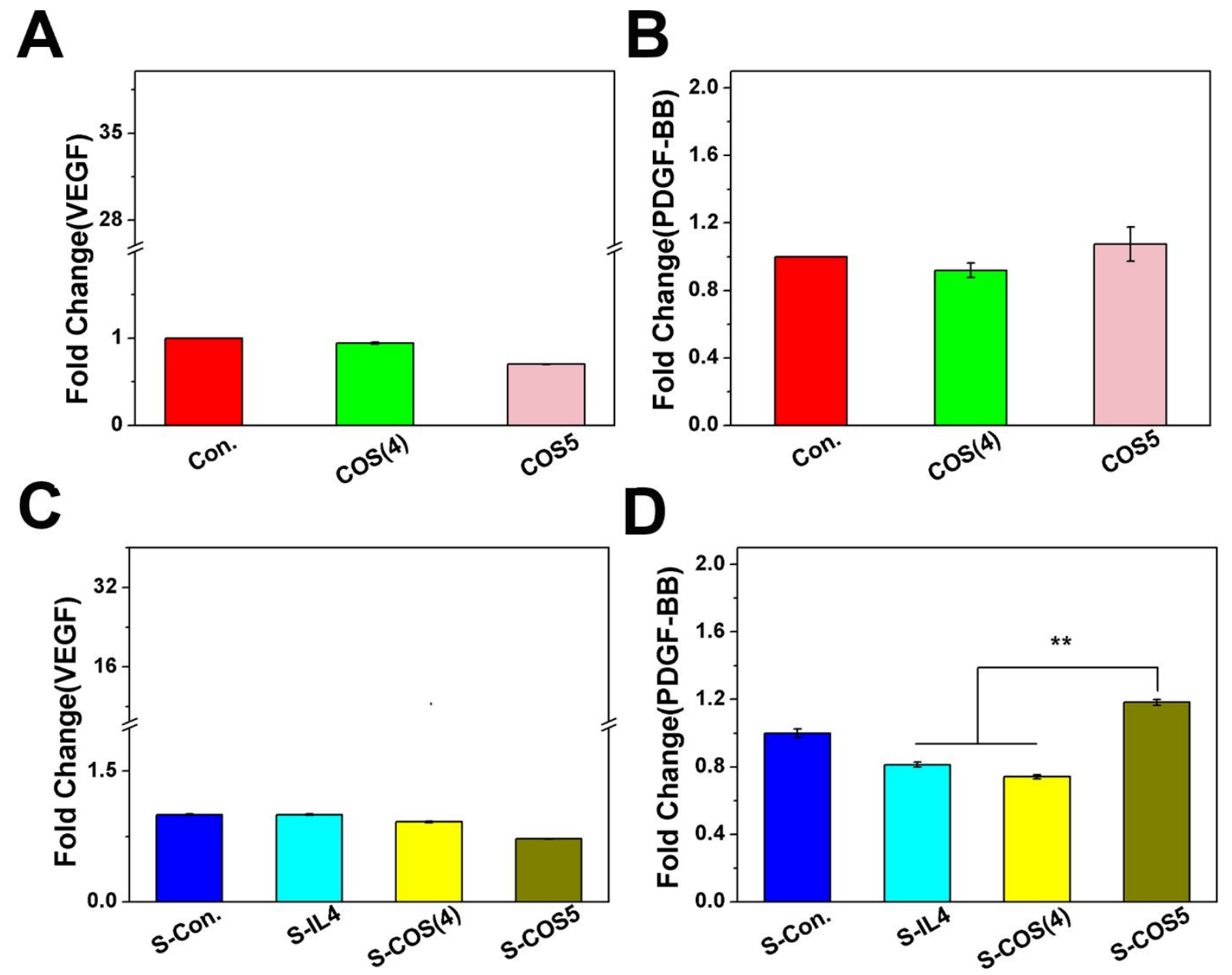

Figure S3. PDGF-BB and VEGF secretion of HUVEC/RAW 264.7 analyzed by ELISA. (A) VEGF and (B) PDGF-BB secretion of HUVECs treated with COS(4) and COS5. (C) VEGF and (D) PDGF-BB cytokines secreted by HUVECs after culturing in conditioned medium of RAW 264.7 S-Con, S-IL4, S-COS(4), and S-COS5. (Statistically significant at ${ }^{*} p<0.05$ and ${ }^{* *} p<0.01$ ) 